\title{
Optimized multicanonical simulations: A proposal based on classical fluctuation theory
}

\author{
J. Viana Lopes \\ Centro de Física do Porto and Departamento de Física, Faculdade de Ciências, Universidade do Porto, 4169-007 Porto, Portugal \\ and Departamento de Física, Instituto Superior de Engenharia, Instituto Politécnico do Porto, Porto, Portugal \\ Miguel D. Costa and J. M. B. Lopes dos Santos \\ Centro de Física do Porto and Departamento de Física, Faculdade de Ciências, Universidade do Porto, $4169-007$ Porto, Portugal \\ R. Toral \\ Instituto Mediterráneo de Estudios Avanzados IMEDEA (CSIC-UIB), \\ Edificio Mateu Orfila, Campus UIB, E-07122 Palma de Mallorca, Spain
}

(Received 26 May 2006; published 5 October 2006)

\begin{abstract}
We propose a recursive procedure to estimate the microcanonical density of states in multicanonical Monte Carlo simulations which relies only on measurements of moments of the energy distribution, avoiding entirely the need for energy histograms. This method yields directly a piecewise analytical approximation to the microcanonical inverse temperature $\beta(E)$ and allows improved control over the statistics and efficiency of the simulations. We demonstrate its utility in connection with recently proposed schemes for improving the efficiency of multicanonical sampling, either with adjustment of the asymptotic energy distribution or with the replacement of single spin flip dynamics with collective updates.
\end{abstract}

DOI: 10.1103/PhysRevE.74.046702

PACS number(s): 02.70.Rr, 64.60.Cn, 75.10.Hk, 05.10.Ln

\section{INTRODUCTION}

Over the past decade, the use of Monte Carlo methods [1] has broken the boundaries of statistical physics and has become a widely used computational tool in fields as diverse as chemistry, biology, and even sociology or finance.

Despite the enormous success of the well-known Metropolis importance sampling algorithm, its narrow exploration of the phase space and characteristic convergence difficulties motivated a number of different approaches. First, the techniques for harvesting useful information from the statistical data obtained at a given temperature were improved in order to extrapolate the results to a larger temperature range and, therefore, reduce the number of required independent simulations $[2,3]$. Secondly, cluster update algorithms were proposed [4-6] in order to overcome critical slowing down. Finally, the requirement of constant temperature was lifted, allowing the system to explore a wider range of the energy spectrum. In simulated tempering, the temperature becomes a dynamical variable, which can change in the Markov process [7]; parallel tempering uses several replicas of the system running at different temperatures and introduces the swapping of configurations between the various Markov chains [8-10]. Multicanonical methods [11,12] replace the Boltzmann distribution for the asymptotic probability of sampling a given state of energy $E_{i}, p_{i} \propto \exp \left(-\beta E_{i}\right)$, by $p_{i} \propto 1 / n\left(E_{i}\right)$, the inverse of the microcanonical density of states. This ensures that every energy value is sampled with equal probability and results in a flat histogram in the distribution of energies.

The first major obstacle to the multicanonical approach is the obvious difficulty of accessing the true density of states of a given system; several clever algorithms have been proposed to this end $[11,13,14]$. Nevertheless, even in cases where the true density of states is known a priori, recent studies $[15,16]$ have shown that very long equilibration times can remain a serious concern. Furthermore, the number of independent samples is strongly dependent on energy, making error estimation rather tricky [17]. This has led some authors [18] to question the reliability of the results obtained by multicanonical methods in spin glass models at low temperatures. To address these issues, the requirement of a perfectly flat histogram was also lifted [19], sacrificing the equal probability of sampling each energy in favor of minimizing tunneling times, defined as the round trip time between two specified energies (most commonly, between the ground state and the maximum of the density of states).

In this paper we propose a variation of the algorithm to estimate the density of states. The main feature of our proposal (that we name "CFP" since it is based on classical fluctuation theory) is that it does not use histograms, instead relying entirely on measurements of cumulants of the energy distribution at each stage of the simulation to build a piecewise analytical approximation to the statistical entropy $S(E)=\ln [n(E)]$ and inverse temperature $\beta(E)=d S(E) / d E$. We find that the time required to explore the entire energy spectrum scales more favorably with system size than histogram based methods. The method is easy to apply in systems with continuous or discrete energy spectrum, can be quite naturally adapted to running a simulation in a chosen energy range, and accommodates without difficulty tunneling times, optimization schemes and cluster update methods.

The paper is organized as follows. In Sec. II we describe the new method and compare its performance with histogram based methods. In Sec. III we show that the optimization scheme proposed recently in Ref. [19] can be applied during the process of estimating the density of states, still avoiding histograms, and with significant efficiency gains. In Sec. IV, we demonstrate the usefulness of the analytical approximation of $\beta(E)$ in generalizing Wolff's cluster algorithm [5] to multicanonical simulations. This generalization maintains an 

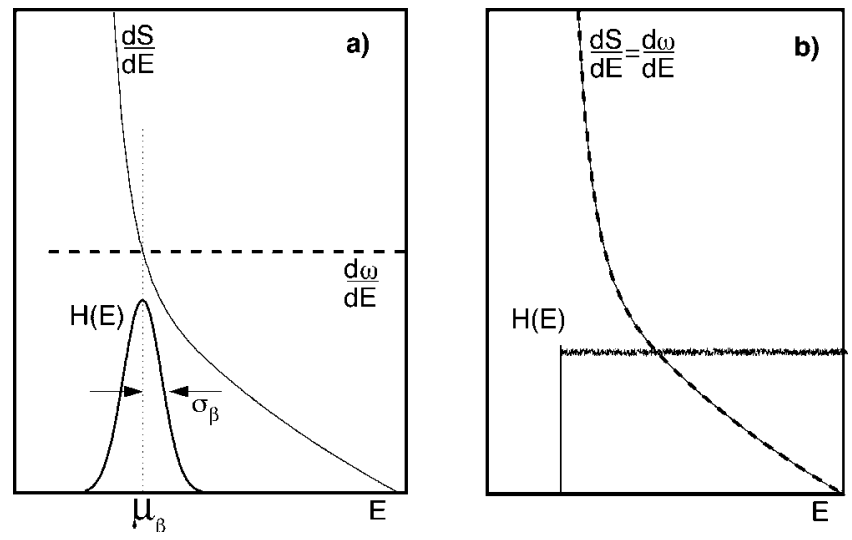

FIG. 1. Schematic representation of the true derivative of the entropy $S(E)$ and two different choices for the derivative of $\omega(E)$, with the corresponding energy probability distributions. In panel (a), the Metropolis approach, where $\omega(E)=\beta E$ and, in panel (b), the ideal multicanonical approach with $\omega(E)=S(E)$.

acceptance probability still very close to unity, growing large clusters at low temperatures and small ones at higher temperatures. The optimization procedure reported in Ref. [19] is also implemented for this cluster dynamics. Finally, the main conclusions are summarized in Sec. V. The CFP algorithm has already been applied with success to both discrete (Ising models on regular lattices and small world networks, Ising spin glasses) [20] and continuous models $(X Y$ and Heisenberg models, with both short- and long-range interactions, namely, dipolar interactions) [21], but this is a systematic presentation.

\section{THE CFP METHOD}

\section{A. Proposal}

The usual way of ensuring a general asymptotic distribution $p_{i} \propto \exp \left[-\omega\left(E_{i}\right)\right]$ is to use a Markov chain algorithm in which the transition probability to go from state $i$ to state $j$ with respective energies $E_{i}$ and $E_{j}$ is

$$
W_{i j}=\min \left(1, e^{-\Delta \omega}\right)
$$

with $\Delta \omega=\omega\left(E_{j}\right)-\omega\left(E_{i}\right)$. This choice leads to an asymptotic energy distribution probability given by

$$
H(E) \propto \exp [S(E)-\omega(E)],
$$

where the entropy is defined as the logarithm of the (unknown) density of energy states, $S(E)=\ln [n(E)]$. In principle, this relation allows a calculation of the entropy $S(E)$ (up to an irrelevant constant $S_{0}$ ) from a numerical determination of the distribution $H(E)$ for any value of the energy $E$ simply as

$$
S(E)=S_{0}+\omega(E)+\ln H(E) .
$$

However, this is not always efficient for any choice of the function $\omega(E)$. Consider, for example, the widely used Metropolis choice $\omega(E)=\beta E$ (with $\beta=1 / T$, the inverse temperature). In many systems, the resulting distribution $H(E)$ has the shape of a bell curve, usually approximated by a Gaussian distribution (Fig. 1). Since it is very unlikely to generate statistically significant configurations in the tails of the distribution for $H(E)$, the usefulness of the formula (3) is limited to values of $E$ not too far from the mean value $\mu_{\beta}$. "Not too far" means explicitly that the above formula is limited to those values of $E$ such that $\left|E-\mu_{\beta}\right|<\alpha \sigma_{\beta}$ with $\alpha \sim 2-4$. In this case, the mean value $\mu_{\beta}$ and the variance $\sigma_{\beta}^{2}$ of the distribution $H(E)$ satisfy

$$
\left.\frac{d S}{d E}\right|_{\mu \beta}=\beta,\left.\quad \frac{d^{2} S}{d E^{2}}\right|_{\mu \beta}=-\frac{1}{\sigma_{\beta}^{2}} .
$$

A clever choice for $\omega(E)$ can greatly improve the range of values of $E$ for which the formula (3) is useful. For instance, if we were to choose $\omega(E) \propto \ln [n(E)]$ then the resulting distribution $H(E)$ would be constant in $E$ and all the energy values would be sampled with the same frequency. However, it is clear that this ideal multicanonical approach is impossible since $n(E)$ is precisely the function we want to determine.

Some methods [13] were devised to estimate numerically the density of states $n(E)$. For this paper it is relevant to consider the conceptually important scheme developed by Berg and collaborators (a review is given in Ref. [22]). Berg's scheme uses a succession of functions $\omega_{i}(E)$, each one of them giving information on $n(E)$ for a range of energy values. The initial choice $\omega_{0}(E)=0$ (equivalent to a Metropolis choice at infinite temperature, $\beta=0$ ) provides a histogram $H_{0}(E)$ from which one derives an estimation of the entropy $S_{0}(E)$ valid for those values of $E$ visited in a statistically significant way. After this stage, a new simulation is performed with $\omega_{1}(E)=S_{0}(E)$ in the region visited in the previous simulation and 0 elsewhere, from which we obtain an entropy estimate $S_{1}(E)$ valid in another range of energies, and so on. Berg proposes an explicit recursion scheme that allows systematic corrections of $\omega_{i}(E)$ at all visited energies, and ensures the convergence to the true entropy for any energy, so ensuring a flat energy histogram.

This procedure has some limitations. To begin with, the entropy can only be estimated inside the energy range visited in the last simulation and a bad choice for the entropy outside this region can severely limit the exploration of lower energies; secondly, rarely visited energies introduce a large error in the estimated entropy (hence the need for recursion introduced by Berg in order to minimize this error). Furthermore, the fact that one counts visits in each energy implies that, for continuous systems, the energy spectrum must be discretized.

Our approach assumes that thermodynamic functions such as the entropy can be treated as continuous functions of energy, both for discrete and continuous spectra, for not too small systems. More specifically, we make use of the Gaussian approximation

$$
S(E) \approx S\left(\mu_{\beta}\right)+\beta\left(E-\mu_{\beta}\right)-\frac{1}{2 \sigma_{\beta}^{2}}\left(E-\mu_{\beta}\right)^{2}
$$

to propose the sequence for the weight functions $\omega(E)$. The procedure is such that at each recursion step the distribution $H(E)$ is flattened in a region centered around the mean and 


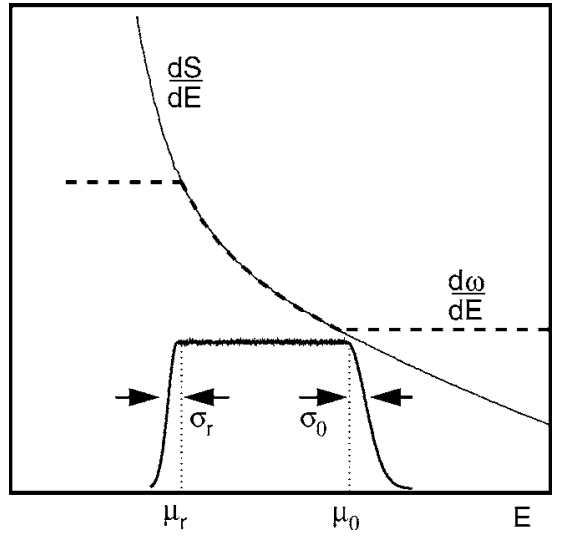

FIG. 2. In the current proposal, the weight function $\omega(E)$ approximates the entropy between two energies $\mu_{r}$ and $\mu_{0}$ and is linear in energy outside this region, $d \omega / d E=\beta_{r}$ for $E<\mu_{r}$ and $d \omega / d E=\beta_{0}$ for $E>\mu_{0}$.

width proportional to the standard deviation of the energy distribution at the previous step.

Our scheme works as follows. After running an initial simulation at inverse temperature $\beta_{0}$, with $\omega_{0}=\beta_{0} E$, we measure the mean value, $\mu_{0}$, and the variance, $\sigma_{0}^{2}$, of the resulting energy distribution. We then modify the weight function to $\omega_{1}(E)$ defined by

$$
\omega_{1}(E)= \begin{cases}\beta_{0} E, & E>\mu_{0}, \\ b_{0}+\beta_{0}\left(E-\mu_{0}\right)-\frac{\left(E-\mu_{0}\right)^{2}}{2 \sigma_{0}^{2}}, & \mu_{1}<E<\mu_{0}, \\ b_{1}+\beta_{1}\left(E-\mu_{1}\right), & E<\mu_{1}\end{cases}
$$

with $\mu_{1}=\mu_{0}-\alpha \sigma_{0}$. In order to ensure continuity of the function and its derivative we determine $b_{0}=\beta_{0} \mu_{0}$, $b_{1}=\beta_{0} \mu_{1}-\frac{\alpha^{2}}{2}, \beta_{1}=\beta_{0}+\alpha / \sigma_{0}$. In a simulation with transition rates $W_{i j}=\min \left(1, e^{-\Delta \omega_{1}}\right)$ we obtain $H(E) \simeq$ const, for energies such that $\mu_{1}<E<\mu_{0}$, while for $E<\mu_{1}, H(E)$ is a "half Gaussian" with maximum at $\mu_{1}$ and half-width $\sigma_{1}$. We now compute $\sigma_{1}^{2}$ as the average of $\left(E-\mu_{1}\right)^{2}$ for $E<\mu_{1}$. We are therefore able to add another branch to $\omega(E)$,

$$
\omega_{2}(E)= \begin{cases}\beta_{0} E, & E>\mu_{0}, \\ b_{0}+\beta_{0}\left(E-\mu_{0}\right)-\frac{\left(E-\mu_{0}\right)^{2}}{2 \sigma_{0}^{2}}, & \mu_{1}<E<\mu_{0}, \\ b_{1}+\beta_{1}\left(E-\mu_{1}\right)-\frac{\left(E-\mu_{1}\right)^{2}}{2 \sigma_{1}^{2}}, & \mu_{2}<E<\mu_{1}, \\ b_{2}+\beta_{2}\left(E-\mu_{2}\right), & E<\mu_{2}\end{cases}
$$

with $\mu_{2}=\mu_{1}-\alpha \sigma_{1}, b_{2}=\beta_{1} \mu_{2}-\frac{\alpha^{2}}{2}$, and $\beta_{2}=\beta_{1}+\alpha / \sigma_{1}$. This process can be repeated with similar recursion relations for the coefficients until we reach the lowest temperature we wish to study. On the iteration of order $r$ the histogram is nearly flat between $\mu_{0}$ and $\mu_{r}$ and half Gaussian below $\mu_{r}$ (Fig. 2). Although the entropy could also be extrapolated to higher values of the energy, using $\omega(E)=\beta_{0} E$ for $E>\mu_{0}$, we effectively restrict the simulation to energies below $\mu_{0}$, apart from a Gaussian tail above this energy.

The fact that $\Delta \omega_{r}(E)=\beta_{r} \Delta E$ in the yet unexplored energy regions, corresponding to Boltzmann sampling, means that we can use all techniques developed for the Metropolis algorithm in order to be confident on the results obtained in this region, before moving on to lower energies. These regions of canonical sampling can also be used to restrict the simulation to a specific temperature range, for instance, around a critical temperature, which can dramatically increase the efficiency and precision of the simulation.

Surely, there are small systematic errors in the estimation of the entropy, but they only influence the flatness of the histogram near the critical temperature for a second order phase transition. There, the method can also be refined by keeping higher order terms in the expansion of the entropy which can be obtained by measuring higher order moments of the energy. We have successfully used an expansion of $S(E)$ up to fourth order terms.

In any case, there is no need for a perfectly flat histogram, since for a given function $\omega(E)$, canonical averages can always be obtained through the relation

$$
\langle A\rangle_{\beta}=\frac{\sum_{i} A_{i} e^{\omega\left(E_{i}\right)-\beta E_{i}}}{\sum_{i} e^{\omega\left(E_{i}\right)-\beta E_{i}}} .
$$

The only requirement on the shape of the histogram is that it is broad enough to include contributions to the desired values of $\beta$.

\section{B. Comparison with histogram based recursion}

We now compare the performance of our proposal (CFP) with that of the histogram based multicanonical recursion as described in Ref. [22]. To this end, we have applied both methods to the two-dimensional nearest-neighbor Ising model, of Hamiltonian

$$
H=-J \sum_{\langle i j\rangle} s_{i} s_{j} .
$$

Except for the method of estimating the density of states (or rather, its logarithm, the entropy), the results for the two methods under comparison were obtained using exactly the same code, specifically with the same number of steps per run. We chose the number of Monte Carlo steps (MCS) in each iteration (run) to increase linearly with the iteration number, $r$, specifically as $r \times 10^{5}$ MCS. This is a rather arbitrary choice which is necessary for comparison purposes. In fact, our proposal allows us to set the number of steps in the yet unexplored energy region as the criteria for moving on to the next run, which, in turn, allows better statistical control over the next estimate for the entropy. For a fair comparison between the two algorithms we choose $\beta_{0}=0$ for CFP and impose $\beta(E)=0$ in the histogram based method for $E>\mu_{0}$. In this way the algorithms only explore the positive temperature region of the spectra.

Figure 3 shows 7 histograms out of 35 iterations on a $64 \times 64$ Ising model, with $r \times 10^{5}$ MCS per run, which was 


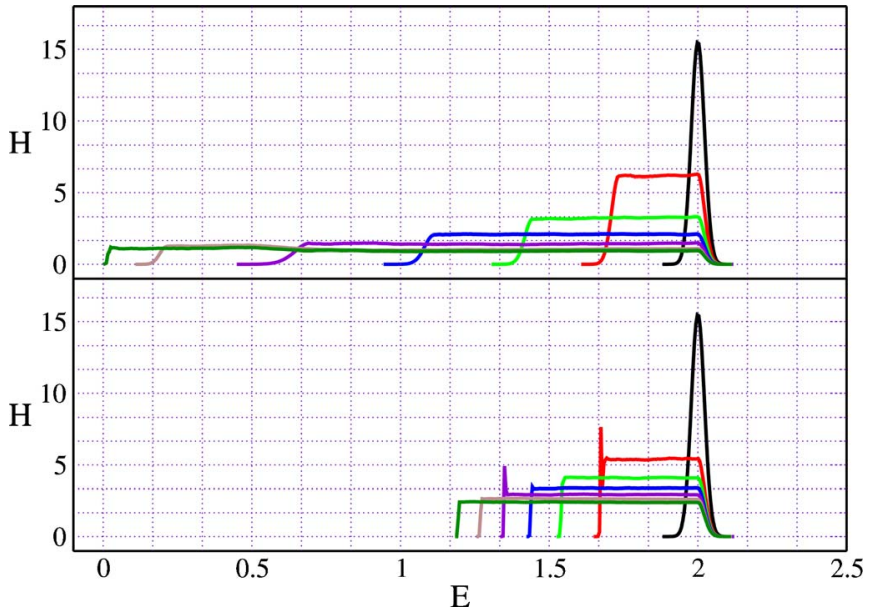

FIG. 3. (Color online) A comparison of the evolution of the multicanonical recursion in the present scheme (upper panel) and the one described in Ref. [22] (lower panel). The simulation was done for an Ising square sample with $L=64$, and, for simplicity, we only show histograms from one out of each five runs.

enough to reach the ground state with our algorithm (we used the value of the parameter $\alpha=2$ ). As shown in the lower panel of that figure, several more runs are required using the histogram based method. In this respect the difference in performance of the two methods is quite remarkable. Moreover, histogram based methods do not provide an estimate for the entropy outside the previously visited energy range. As a result, for energies in the previously unvisited range, $H(E)$ decays as

$$
H(E) \sim \exp [S(E)] \sim \exp \left[-\beta_{r}\left(E_{r}-E\right)\right]
$$

for $E<E_{r}$, where $E_{r}$ is the lowest energy of the previous run and $\beta_{r}=\beta\left(E_{r}\right)$. Our method uses in the same range $\omega(E)=\beta_{r} E+$ const which leaves

$$
H(E) \sim \exp [S(E)-\omega(E)] \sim \exp \left[-\frac{1}{2 \sigma_{r}^{2}}\left(E_{r}-E\right)^{2}\right] .
$$

Since $\sigma_{r}^{2}$ scales linearly with $N$, the added energy range in each iteration scales differently in the two methods. In terms of energy per particle, the added energy range per run, $\Delta \epsilon_{r}$ $=\left|\mu_{r}-\mu_{r-1}\right| / N=\alpha \sigma_{r}$, scales as $\Delta \epsilon_{r} \propto \beta_{r} c_{r}^{1 / 2} N^{-1 / 2}\left(c_{r}\right.$ is the specific heat at temperature $\beta_{r}$ ) in the CFP instead of $\Delta \epsilon_{r}$ $\propto\left(\beta_{r} N\right)^{-1}$ in histogram based methods. In Fig. 4 we plot the visited energy per particle range after $r$ iterations, for various system sizes, as a function of $r / N^{1 / 2}$ for the CFP method and $r / N$ for the histogram based method. The collapse of the curves for the various system sizes shows that the number of runs required to cover the same energy per particle range scales with $\sqrt{N}$ in the current proposal (left panel) and $N$ in histogram based methods (right panel). Hence, although the two methods perform similarly for small systems, the advantage of the CFP method becomes obvious for large $N$ and no amount of fine tuning can disguise this advantage.

Other alternative schemes to Berg's recursion have already been proposed such as Wang-Landau sampling [23],
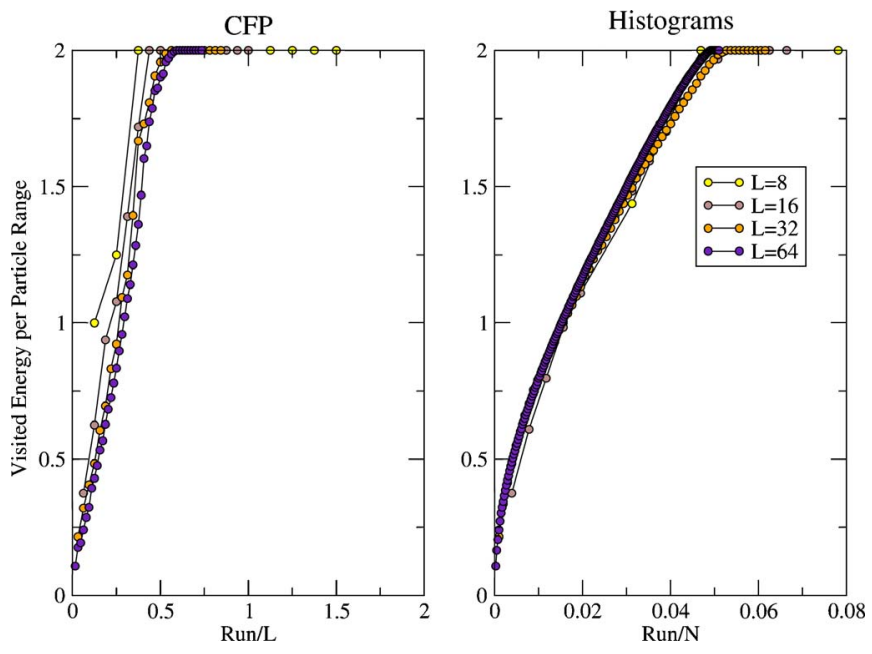

FIG. 4. (Color online) Full range of visited energies $\left(E_{r}-E_{0}\right) / N$, as a function of the system size and number of iterations $r$ for current method (left panel) and for histogram based recursion (right panel); in our moment based method $r$ scales $L=\sqrt{N}$ whereas, with histograms, it scales with $N$.

the histogram by overlapping windows [13], or the transition matrix method [24]. As will be seen shortly, the main advantage of our method is that, unlike these previous methods, it produces an analytic approximation to the microcanonical inverse temperature $\beta(E)$ in an increasing energy range, right from the start of the simulation. That proves an asset in the implementation of procedures designed to overcome the slowing down with system size that affects multicanonical simulations $[15,19,25]$.

\section{OPTIMIZATION OF TUNNELING TIMES}

In a recent publication [19], Trebst, Huse, and Troyer have shown that it is possible to significantly decrease the tunneling times of a multicanonical simulation, by abandoning the requirement of a flat histogram. Our procedure of construction of the statistical entropy is well suited to implement their optimization method, right from the start of the simulation, without having to construct an approximation to $n(E)$ for the entire spectrum.

The procedure proposed in Ref. [19] minimizes the average time required to span the gap between two fixed energies in the spectrum $E_{-}$and $E_{+}$. To achieve this purpose, one must distinguish each energy entry during the simulation according to which of the two energies $E_{-}$or $E_{+}$was visited last. We can thus measure separately $n_{-}(E)$, the number of visits to energy $E$ occurring when the simulation has visited $E_{-}$ more recently than $E_{+}$, and $n_{+}(E)$ for the other way around. To minimize the tunneling times between the two energies $E_{-}$and $E_{+}$one must choose an asymptotic energy distribution that satisfies

$$
H(E) \propto \frac{d f(E)}{d E},
$$

where $f(E)=n_{+}(E) / H(E)$. The implementation proposed in Ref. [19] used the knowledge of the density of states $n(E)$ in 
the whole spectrum to measure the $f(E)$, following with a recursion procedure that converges to the asymptotic distribution which satisfies Eq. (12). In our method of construction of the density of states there are, at each step, two energies which are the current boundaries of the known density states, namely $\mu_{0}$ (that remains fixed) and $\mu_{r}$ (that changes with each run, $r$ ). Therefore, by using $E_{-}=\mu_{r}$ and $E_{+}=\mu_{0}$ we can measure $f(E)$. In the first run, for $\mu_{1}<E<\mu_{0}$, we observe $H_{1}(E) \propto \exp \left[-\omega_{1}(E)\right]$, with $\omega_{1}(E)$ defined in Eq. (6), rather than the optimal distribution $H^{\text {opt }}(E) \propto \exp \left[\ln \left(d f_{1} / d E\right)\right]$. To converge to the optimal distribution [changing the weight alters $f(E)$ ], we use, in the next run, the geometric mean $\sqrt{H_{1} H^{\mathrm{opt}}}$ in the interval $\mu_{1} \leqslant E$ $\leqslant \mu_{0}[19]$ : the weight factor becomes $-\omega_{2}(E)+\phi_{2}(E)$, where

$$
\phi_{2}(E)=\frac{1}{2} \ln \left(\frac{d f_{1}}{d E}\right) \quad \text { for } \mu_{1} \leqslant E \leqslant \mu_{0},
$$

with constant values of $\phi_{2}\left(\mu_{1}\right)$ for $E<\mu_{1}$ and $\phi_{2}\left(\mu_{0}\right)$ for $E>\mu_{0}$. Notice that the correction to the microcanonical temperature $\beta(E)$ is $-d \phi_{2}(E) / d E$ which is zero for $E<\mu_{1}$. This choice ensures the convergence to the criterion of Eq. (12) in the range where the entropy is already known and where $f(E)$ was measured, $\mu_{1}<E<\mu_{0}$, and gives a flat histogram in the region where the entropy was estimated by calculating moments of the Gaussian tail, i.e., $\mu_{2}<E<\mu_{1}$. This procedure is iterated in the following runs with $\phi_{r}(E)$ defined as

$$
\phi_{r}(E)=\frac{1}{2}\left[\phi_{r-1}+\ln \left(\frac{d f_{r-1}}{d E}\right)\right], \quad \mu_{r-1} \leqslant E \leqslant \mu_{0}
$$

with constant values $\phi_{r}\left(\mu_{r-1}\right)$ for $E<\mu_{r-1}$ and $\phi_{r}\left(\mu_{0}\right)$ for $E>\mu_{0}$. To extract the numerical derivative of $f(E)$, avoiding the difficulties of the fluctuations in histogram entries, we use the natural scale afforded by $\sigma_{\beta}$, and calculate $d f / E$ as

$$
\left.\frac{d f}{d E}\right|_{\mu_{\beta}}=\frac{\left\langle f(E)\left(E-\mu_{\beta}\right)\right\rangle_{\beta}}{\sigma_{\beta}^{2}} .
$$

This quantity can be calculated using histograms or, using the definition of $f(E)$, through the relation

$$
\left.\frac{d f}{d E}\right|_{\mu_{\beta}}=\frac{\sum_{i}^{+}\left(E_{i}-\mu_{\beta}\right) e^{\omega\left(E_{i}\right)-\beta E_{i}}}{\sum_{i} e^{\omega\left(E_{i}\right)-\beta E_{i}}},
$$

where the superscript + denotes a summation restricted to sampled states which contribute to $n_{+}(E)$.

In Fig. 5 we plot the average tunneling time, divided by $(N \ln N)^{2}$, for two different system sizes, as a function of the run number of the multicanonical recursion divided by $\sqrt{N}$; simulations with the same horizontal coordinate correspond to the same energy per particle range. Curves (a) correspond to the situation without optimization, and the average tunneling times vary faster than $(N \ln N)^{2}$; the two sizes do not collapse to a single curve. In case (c), with the optimization carried out while the density of states is being determined, the curves for the two system sizes track each other. If the optimization correction is only performed after

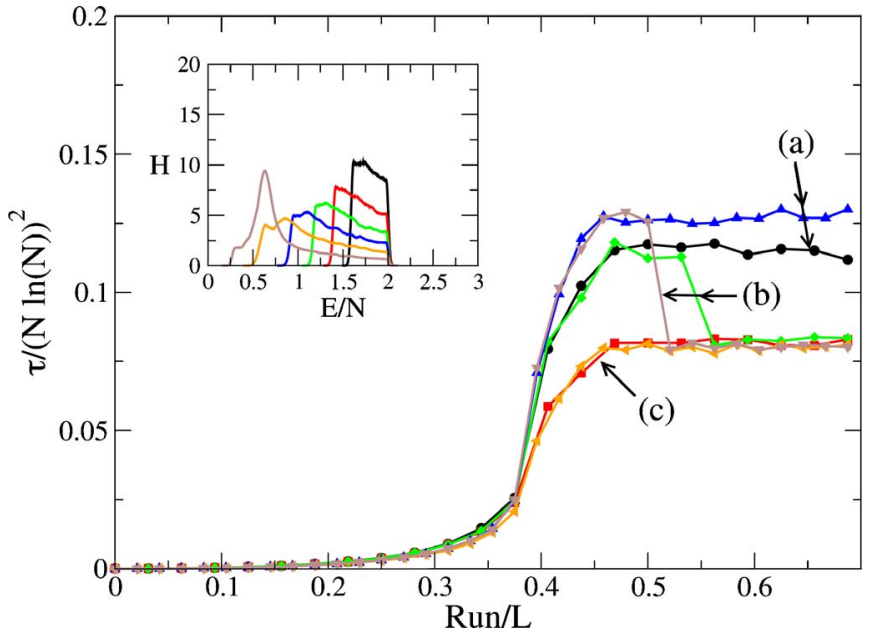

FIG. 5. (Color online) Tunneling time $[\text { scaled by }(N \ln N)]^{2}$, for system sizes $N=48 \times 48$ and $N=64 \times 64$, as an function of the recursion run divided by $L$ which is proportional to the energy per particle range. (a) CFP without optimization; (b) for an application of the optimization procedure only after an estimation of density of states in the chosen range of temperatures; (c) our implementation of the optimization. The inset shows the histograms obtained for the sample with $L=64$ in several runs during the exploration of the energy spectrum with optimization.

full exploration of the energy spectrum, curve (b), there is no further gain in tunneling time, as the average tunneling times of (b) merge with curve (c). This observation clearly supports our suggestion that optimization can be implemented while the density of states is being constructed. In this fashion, it not only reduces tunneling times when $n(E)$ is known, but also speeds up the actual calculation of $n(E)$. As found in Ref. [19], the inset shows a strong signature of the optimizing procedure in the critical energy where the diffusivity is low.

\section{CLUSTER DYNAMICS}

An alternative way to improve the efficiency of multicanonical simulations consists in changing the specific dynamics of the Markov chain, i.e., the algorithms used to propose and accept configuration moves. In canonical ensemble simulations, cluster update algorithms such as Wolff's [5], Swendsen and Wang's [6], or Niedermeyer's [4] have proved very effective in overcoming critical slowing down of correlations. Several proposals have been presented to generalize cluster update approaches in multicanonical ensemble simulations either using spin-bond representations of the partition function [25-28] or cluster building algorithms based on alternative ways of computing the microcanonical temperature $\beta(E)[29,30]$.

Wolff's cluster algorithm [5] provides a clever way of growing a cluster of parallel spins which can be flipped with probability 1 , and still maintain the required Boltzmann asymptotic distribution. This remarkable possibility is intimately related to the fact that $\omega(E)$ is linear in energy $\omega(E)=\beta E$ in a Metropolis simulation. 


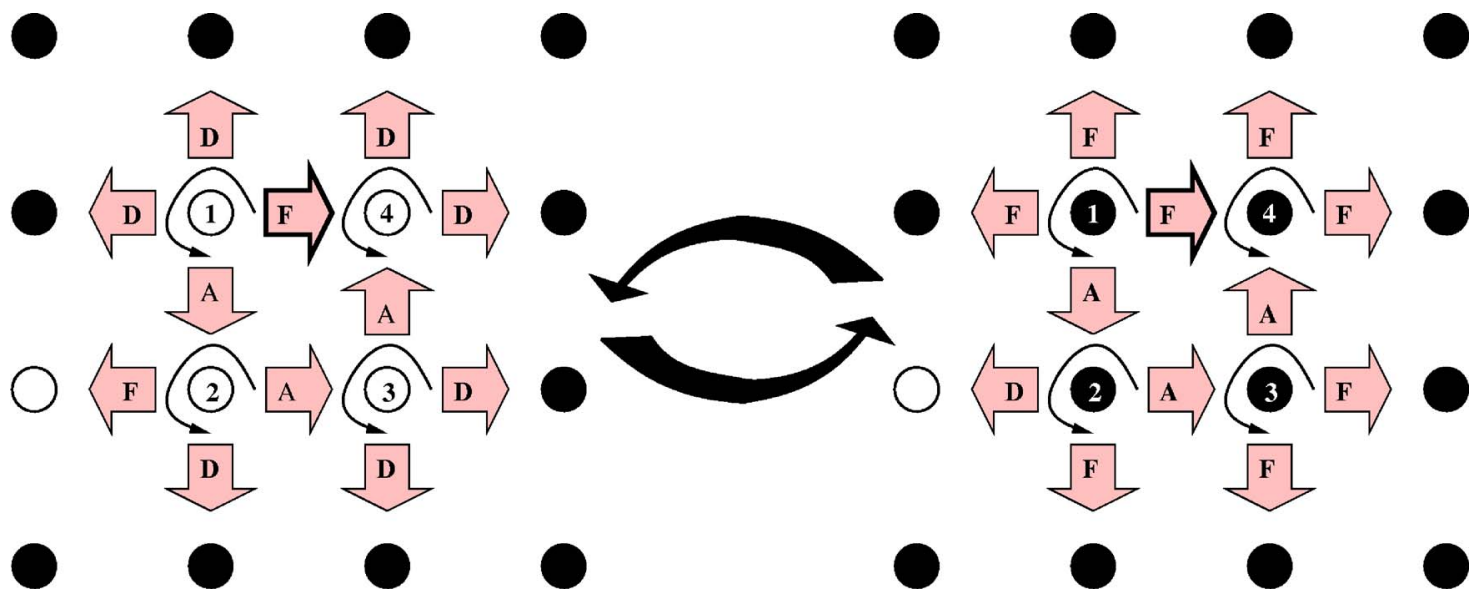

FIG. 6. (Color online) This scheme shows, on the left, how, starting from a given spin (marked as 1), the bonds to neighboring spins are inspected and marked according to the algorithm. On the right are shown the resulting configuration and the way the initial state can be reached from it.

In a multicanonical simulation, each step of the corresponding Markov chain occurs with the same probability as that of a canonical ensemble simulation, with an effective temperature $\beta_{i}$ chosen as $\beta_{i}=\beta\left(E_{i}\right), E_{i}$ being the energy of the current configuration; hence the designation "multicanonical." With this in mind, the simplest way of implementing cluster dynamics in a multicanonical simulation is to use $\beta_{i}=\left.\frac{d \omega(E)}{d E}\right|_{E=E_{i}}$ to grow a cluster exactly as proposed in Wolff's algorithm. However, since the reverse path implies a different value of $\beta, \beta_{j}=\beta\left(E_{j}\right)$, where $E_{j}$ is the energy of the next configuration in the chain, we must include an acceptance probability to ensure detailed balance.

In Fig. 6 we illustrate a move involving the flipping of four spins (labeled 1 to 4 ) on the left, and the reverse move on the right. The site marked with the number 1 has been chosen with uniform probability. If a bond connects spin 1 to a neighboring spin parallel to 1 it is added to the cluster with a probability $p_{i}$ and rejected with probability $1-p_{i}$. This step is then repeated for the neighbors of the initial spin which were added to the cluster, until the process stops and there are no further bonds that can be aggregated to the cluster. The probability of generating a cluster with $n_{a}$ accepted bonds, in which $n_{r}$ bonds to spins parallel to the initial one were inspected and rejected, is given by

$$
G_{i \rightarrow f}=p_{i}^{n_{a}}\left(1-p_{i}\right)^{n_{r}} .
$$

It is important to note that $n_{r}$ includes a number of rejected bonds that now link spins inside the cluster (such as the bond from spin 1 to spin 4 in Fig. 6). We write $n_{r}=n_{p}+n_{f}$, where $n_{p}$ counts the number of such bonds and $n_{f}$ is the number of bonds from spins in the cluster to parallel spins outside the cluster. This distinction is important when considering the reverse move.

The difference in energy between the final and initial configuration is determined only by the frontier of the cluster; it is given as $\Delta E=-2 J\left(n_{d}-n_{f}\right)$ where $n_{d}$ is the number of bonds to spins opposite to the spins in the cluster. Let us now consider the reverse move, which requires us to select the same cluster in the same order with the spins now reversed with respect to the original state.

Referring once again to Fig. 6, one can see that the $n_{a}$ bonds that were accepted in the direct move (left) with probability $p_{i}$ must be accepted in the reverse move (right), each with probability $p_{j}$; the $n_{d}$ bonds to opposite spins in the direct move now connect to spins parallel to those in the cluster and must be rejected with probability $\left(1-p_{j}\right)$; the bonds to spins that were rejected in the direct move $\left(n_{f}\right)$ are, in the reverse process, bonds to opposite spins and are, hence, rejected with probability 1 ; finally the $n_{p}$ bonds that were rejected but link spins inside the cluster must now also be rejected. In other words, for the direct move

$$
G_{i \rightarrow f}=p_{i}^{n_{a}}\left(1-p_{i}\right)^{n_{f}}\left(1-p_{i}\right)^{n_{p}}
$$

while, for the reverse process,

$$
G_{f \rightarrow i}=p_{j}^{n_{a}}\left(1-p_{j}\right)^{n_{d}}\left(1-p_{j}\right)^{n_{p}}
$$

Wolff's algorithm corresponds to choosing $p_{i}=p_{j}=1-\exp (-2 J \beta)$ which implies that

$$
\frac{G_{f \rightarrow i}}{G_{i \rightarrow f}}=\left(1-p_{i}\right)^{n_{d}-n_{f}}=e^{\beta \Delta E} .
$$

The detailed balance condition for Boltzmann's equilibrium distribution is obtained for an acceptance probability of 1 for flipping the cluster. To ensure an asymptotic distribution proportional to $\exp [-S(E)]$, the detailed balance requires an acceptance probability given by

$$
A_{i \rightarrow f}=\min \left(1, \frac{G_{f \rightarrow i}}{G_{i \rightarrow f}} e^{-\Delta S(E)}\right) .
$$

If we choose $p_{i}=1-e^{-2 \beta_{i} J}$ where $\beta_{i}=\beta\left(E_{i}\right)$, we find that this acceptance probability remains close to 1 for most of the energy range (see Fig. 7), falling only at very low temperatures. This behavior is in strong contrast with the one for single spin flip dynamics, where the acceptance rate is only 1 


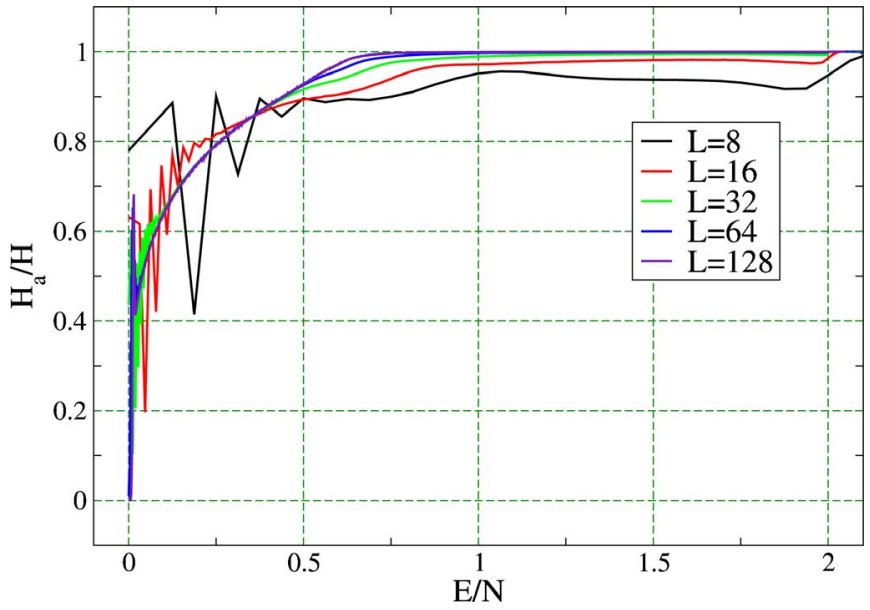

FIG. 7. (Color online) Acceptance ratio of the Wolff 's cluster algorithm, with a microcanonical $\beta(E)$, for the $2 \mathrm{D}$ ferromagnetic Ising model. In the paramagnetic phase the acceptance ratio grows to 1 with the increase of system size; in the ferromagnetic phase the acceptance ratio tends to a finite value, smaller than 1 , as $N$ grows.

at the maximum of the density of states. If the inverse temperatures of the initial and final states are close, $\beta_{i} \approx \beta_{j}$, the acceptance rate becomes

$$
\begin{aligned}
A_{i \rightarrow f} & \approx \min \left(1,\left(1-p_{i}\right)^{n_{f}-n_{d}} e^{-\Delta S(E)}\right) \\
& =\min \left(1, e^{\beta_{i} \Delta E} e^{-\Delta S(E)}\right),
\end{aligned}
$$

where we used $\Delta E=-2 J\left(n_{d}-n_{f}\right)$ to obtain the second expression. For small energy differences, $\Delta S \approx \beta_{i} \Delta E$ and the acceptance rate becomes close to unity.

In the case of the 2D ferromagnetic Ising model, the average excitation energy $\Delta E$ is of $O(\sqrt{N})$ for energies below $\mu_{c}\left(\mu_{c}=\mu_{\beta_{c}}\right.$ where $\beta_{c}$ is the inverse critical temperature), of $O(1)$ for energies above $\mu_{c}$, with a crossover between these regimes in the neighborhood of $\mu_{c}$ (Fig. 8). This behavior reflects a huge difference with respect to single spin flip dynamics (SSF), where $\Delta E$ is always of $O(1)$. A similar behavior exists for the number of spins $n_{v}$ that are inspected in each call of the Markov chain: $n_{v} \sim O(1)$ for $E>\mu_{c}$ and $n_{v} \sim O(N)$ for $E<\mu_{c}$. This two regime behavior introduces an additional complexity in this method. In particular, the tunneling time measured in Markov chains calls no longer scales as the computational time with system size, since Markov chain calls can take a computational time of order $O(N)$. We therefore redefine the time scale so that a Markov chain call in a state of energy $E$ corresponds to a time span of $n_{v}(E)$, the average number of inspected spins in the cluster buildup process.

We now consider the system's coarse grained random walk in energy space. When the energy is close to $E$, the mean square energy change in $M$ Markov chain calls is

$$
\left\langle\Delta E^{2}\right\rangle_{M}=\left\langle\Delta E^{2}\right\rangle \times M
$$

and this occurs in a time $\tau_{M}=M \times n_{v}(E)$. With time measured in this way, the diffusivity of this random walk in energy space is
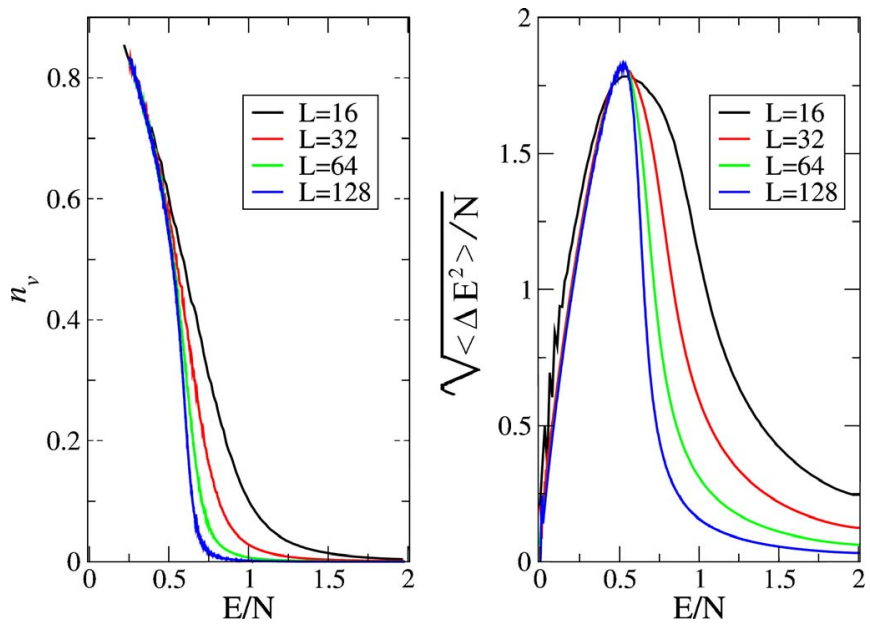

FIG. 8. (Color online) Two regime behavior of the Wolff's cluster algorithm for the 2D ferromagnetic Ising model, with a microcanonical temperature $\beta(E)$. The left panel represents the equal energy average of the fraction of visited spins $n_{v}$ during the cluster growing. In the ferromagnetic phase the number of visited spins is of the order of $O(N)$ and in the paramagnetic phase is of $O(1)$. The right panel represents the equal energy average of the excitations $\Delta E$. In the paramagnetic phase $\sqrt{\left\langle\Delta E^{2}\right\rangle}$ becomes independent of the system size, while in the ferromagnetic phase it scales as $\sqrt{N}$.

$$
D(E) \propto \frac{\left\langle\Delta E^{2}\right\rangle}{n_{v}(E)} .
$$

On the other hand, the probability that the system is at energy $E$ is proportional to

$$
H_{v}(E)=H(E) \times n_{v}(E),
$$

since each visit to energy $E$ lasts a time $n_{v}(E)$. The probability current is given, quite generally, by

$$
j=\rho(E) V(E)-\frac{d}{d E}[D(E) \rho(E)],
$$

where, in equilibrium, $j=0$, and

$$
\rho(E)=\rho_{0}(E) \equiv \frac{H_{v}(E)}{\int d E H_{v}(E)} .
$$

$V(E)$ is a bias field, in general nonzero, which, together with $D(E)$, determines the equilibrium distribution.

It can be shown [19,31] that the tunneling times of a random walk with a given diffusivity $D(E)$ can be minimized with an optimal choice of bias field $V(E)$. The corresponding equilibrium distribution is given by

$$
\rho_{0}(E)=\frac{1}{\sqrt{D(E)}} .
$$

Using Eqs. (25) and (26), this condition becomes 

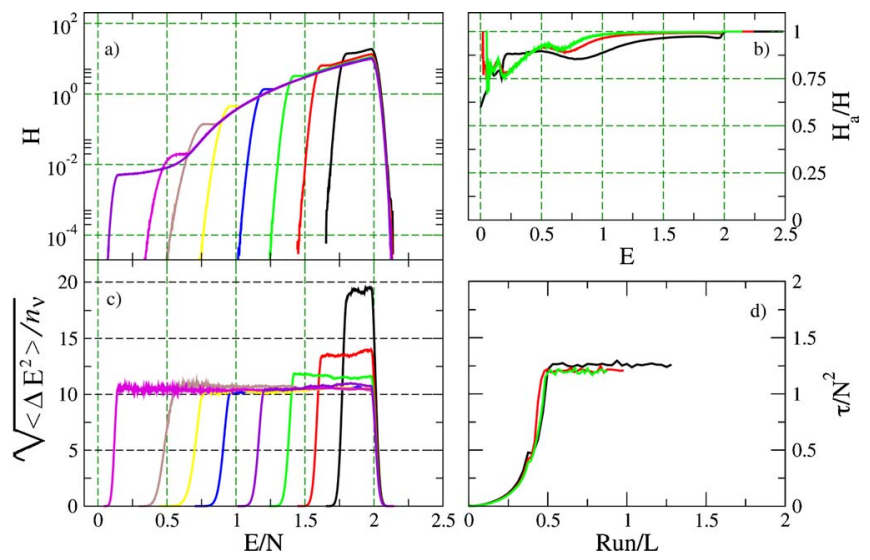

FIG. 9. (Color online) Results of the CFP recursion to construct a histogram given by Eq. (30). (a) Histogram of the simulation for several steps of the CFP recursion, (b) acceptance ratio, (c) $[H(E)$ $\left.\times \sqrt{n_{v}(E)\left\langle\Delta E^{2}\right\rangle}\right]$, and (d) tunneling time (scaled by $N^{2}$ ) versus the run of the recursion (scaled by $L$ ).

$$
H(E) \propto \frac{1}{\sqrt{\left\langle\Delta E^{2}\right\rangle n_{v}(E)}} .
$$

This variation of $\ln [H(E)]$, relative to a flat histogram, is of order $O(\ln (N))$, and, therefore, histograms remain broad, covering the entire spectrum, but are no longer flat as shown in panel (a) of Fig. 9. On panel (d) it is shown that the tunneling time for this simulation scale has $N^{2}$ as expected from a simple diffusion.

The optimization procedure presented in Ref. [19], in the context of $\mathrm{N}$-fold way dynamics, is closely related to this one (but not identical) and leads to a choice

$$
H(E) \propto \frac{1}{n_{v}(E)} \frac{d f}{d E},
$$

where $f(E)$ was defined above. We find only a marginal improvement in tunneling times with respect to the case of a histogram defined by Eq. (30). These two procedures will be compared in another publication [31].

\section{CONCLUSION}

We have proposed a method to build the density of states in a multicanonical simulation. The method is based on the calculation of moments of the energy distribution. It avoids the use of histograms and can just as easily be implemented for continuous systems. It leads to a piecewise analytic approximation to the microcanonical inverse temperature $\beta(E)$. This fact could also be of advantage in multicanonical molecular dynamics simulations [32] where this parametrization could lead to a better way of scaling the forces for multicanonical sampling.

In any stage of the simulation there are two well defined energies, $E_{-}$and $E_{+}$, that limit the range in which $\beta(E)$

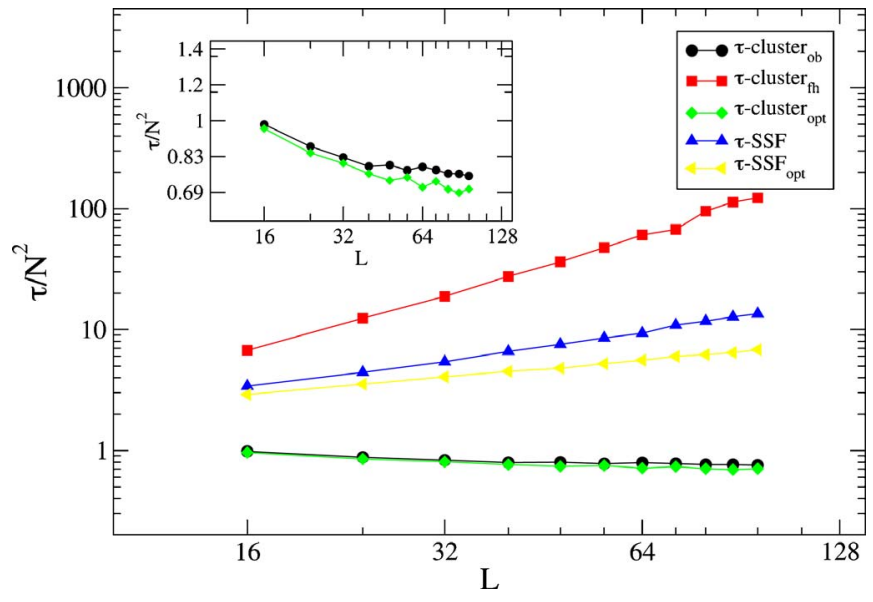

FIG. 10. (Color online) Scaling of tunneling time with system size in various broad histogram methods: (SSF) straightforward multicanonical simulation with single spin flip dynamics, $\left(\mathrm{SSF}_{\mathrm{opt}}\right)$ optimized single spin flip dynamics, $\left(\right.$ cluster $_{\mathrm{OB}}$ ) optimized bias for the measured $D(E)$, ( cluster $_{\text {opt }}$ ) optimized ensemble with cluster dynamics, (cluster $_{\mathrm{FH}}$ ) flat histogram with cluster dynamics.

is known. Therefore the method can be applied without difficulty to a predefined temperature range such as a neighborhood of a critical temperature.

We have also demonstrated the usefulness of this method in the implementation of various optimization schemes that render the simulation more efficient. In Fig. 10 we sum up the results we obtained for the scaling of tunneling times with the system size. In general the scaling of the average tunneling time is $\tau \sim N^{2+z}$. In a straightforward multicanonical simulation with a SSF dynamics, $z=0.39$. Using the optimization procedure of Ref. [19] we confirm that $\tau \sim(N \ln N)^{2}$. For a generalization of Wolff's cluster method for the multicanonical ensemble we found a biased random walk in energy with $z=0.82$. We proposed a method for reducing tunneling time of cluster update simulations which adjusts the bias of the random walk in energy space. In this case, (cluster ${ }_{\mathrm{OB}}$ ) and also for optimized ensemble simulation with cluster algorithm, proposed in Ref. [19] (cluster $_{\text {opt }}$ ), the results are compatible with $z=0$. In the $\left(\operatorname{cluster}_{O B}\right)$ method, however, one avoids the necessity to calculate of the derivative of $f(E)$, required for the (cluster ${ }_{\text {opt }}$ ) method of Ref. [19]. In terms of actual computer time, we also found that the amplitudes of the scaling laws are considerably smaller for the optimized cluster method, than for SSF dynamics.

\section{ACKNOWLEDGMENTS}

The authors would like to thank J. Penedones for very helpful discussions. This work was supported by FCT (Portugal) and the European Union, through POCTI (QCA III). Two of the authors, J.V.L. and M.D.C., were supported by FCT Grant Nos. SFRH/BD/1261/2000 and SFRH/BD/ 7003/2001, respectively. R.T. was supported by the Spanish Government and FEDER (EU) through Project Nos. FIS2004-5073, FIS2004-953. 
[1] M. E. J. Newman and G. T. Barkema, Monte Carlo Methods in Statistical Physics (Oxford University Press, Oxford, 1999).

[2] A. M. Ferrenberg and R. H. Swendsen, Phys. Rev. Lett. 61, 2635 (1988).

[3] A. M. Ferrenberg and R. H. Swendsen, Phys. Rev. Lett. 63, 1195 (1989).

[4] F. Niedermayer, Phys. Rev. Lett. 61, 2026 (1988).

[5] U. Wolff, Phys. Rev. Lett. 62, 361 (1989).

[6] R. H. Swendsen and J. S. Wang, Phys. Rev. Lett. 58, 86 (1987).

[7] E. Marinari and G. Parisi, Europhys. Lett. 19, 451 (1992).

[8] K. Hukushima and K. Nemoto, J. Phys. Soc. Jpn. 65, 1604 (1996).

[9] M. C. Tesi, E. J. J. VanRensburg, E. Orlandini, and S. G. Whittington, J. Stat. Phys. 82, 155 (1996).

[10] H. Hagura, N. Tsuda, and T. Yukawa, Phys. Lett. B 418, 273 (1998).

[11] B. A. Berg and T. Celik, Phys. Rev. Lett. 69, 2292 (1992).

[12] J. Lee, Phys. Rev. Lett. 71, 211 (1993).

[13] G. Bhanot, R. Salvador, S. Black, P. Carter, and R. Toral, Phys. Rev. Lett. 59, 803 (1987).

[14] F. Wang and D. P. Landau, Phys. Rev. Lett. 86, 2050 (2001).

[15] P. Dayal, S. Trebst, S. Wessel, D. Wurtz, M. Troyer, S. Sabhapandit, and S. N. Coppersmith, Phys. Rev. Lett. 92, 097201 (2004).

[16] M. D. Costa, J. Viana Lopes, and J. M. B. Lopes dos Santos, Europhys. Lett. 72, 802 (2005).
[17] J. Viana Lopes and J. M. B. Lopes dos Santos (unpublished). [18] E. Marinari, G. Parisi, F. Ricci Tersenghi, and F. Zuliani, J. Phys. A 34, 383 (2001).

[19] S. Trebst, D. A. Huse, and M. Troyer, Phys. Rev. E 70, 046701 (2004).

[20] J. Viana Lopes, Yu. G. Pogorelov, J. M. B. Lopes dos Santos, and R. Toral, Phys. Rev. E 70, 026112 (2004).

[21] M. D. Costa, Master's thesis, Faculdade de Ciências, Universidade do Porto, 2001.

[22] B. A. Berg, Comput. Phys. Commun. 153, 397 (2003).

[23] F. Wang and D. P. Landau, Phys. Rev. Lett. 86, 2050 (2001).

[24] J. S. Wang, T. K. Tay, and R. H. Swendsen, Phys. Rev. Lett. 82, 476 (1999).

[25] Y. Wu, M. Korner, L. Colonna Romano, S. Trebst, H. Gould, J. Machta, and M. Troyer, Phys. Rev. E 72, 046704 (2005).

[26] W. Janke and S. Kappler, Phys. Rev. Lett. 74, 212 (1995).

[27] W. Janke and S. Kappler, Nucl. Phys. B 42, 876 (1995).

[28] C. Yamaguchi and N. Kawashima, Phys. Rev. E 65, 056710 (2002).

[29] S. Reynal and H. T. Diep, Comput. Phys. Commun. 169, 243 (2005).

[30] S. Reynal and H. T. Diep, Phys. Rev. E 72, 056710 (2005).

[31] J. Penedones, J. Viana Lopes, and J. M. B. Lopes dos Santos (unpublished).

[32] N. Nakajima, H. Nakamura, and A. Kidera, J. Phys. Chem. B 101, 817 (1997). 